•编者按・

\title{
保护生物学、保护生态学与生物多样性科学
}

\author{
马克平 \\ (中国科学院植物研究所植被与环境变化国家重点实验室, 北京 100093)
}

\section{Conservation Biology, Conservation Ecology and Biodiversity Science}

\section{Keping Ma}

State Key Laboratory of Vegetation and Environmental Change, Institute of Botany, Chinese Academy of Sciences, Beijing 100093

在查阅生物多样性方面的文献时，经常会遇到 3个学科, 即保护生物学、保护生态学和生物多样性 科学。三者的关系如何? 各自具有怎样的特点和发 展过程?

为了更好地理解 3 个学科的产生背景和特点, 需要回顾一下始于 19 世纪中叶的保护运动 (conservation movement)的历史。最早可以追溯到英 属印度时期的公民义务(civic duty)。主要包括三点: (1)人类活动, 特别是殖民者疯狂掠夺其殖民地的自 然资源, 对环境造成了严重破坏; (2)人类负有为后 代保护环境的公民义务; (3)科学的方法应该用于履 行公民义务。为此, 英属印度成立了森林部 (Stebbing,1922), 并相继启动了若干森林保护项目, 且逐步影响到其他殖民地开展森林保护行动。当时 在印度工作的英国科学家发布了一份报告, 认为森 林破坏与降雨和水源的减少以及当地人的贫困有 直接关系, 并敦促在印度次大陆建立广泛的、由专 业林业人员管理的森林保护地(Primack等, 2014)。 19世纪晚期, 由于农业的扩张和狩猎工具的进步, 野生动物大量减少, 对英国文化和生态都十分重要 的动物, 如大鸨 (Otis tarda)、海雕 (Haliaeetus albicilla)和大海雀(Pinguinus impennis)等相继灭绝。 严峻的现实激发了英国对野生动物的保护热情, 英 国海鸟保护法案于1869年通过, 成为世界上第一部 自然保护法(Baeyens \& Martinez, 2008)。英国皇家鸟 类保护协会于1889年成立(http://www.rspb.org.uk/), 国家名胜古迹信托公司于1895年成立(Primack等, 2014)。
美国也是自然保护运动的引领者之一。亨利.大 卫.梭罗(H. D. Thoreau)是自然保护倡导者之一, 他 在瓦尔登湖畔自己搭建的小木屋中原生态地生活 了两年多, 其代表作《瓦尔登湖》一书于1854年出 版, 不仅影响了当时的年轻人和环保主义者, 今天 仍在全球自然保护领域具有积极影响。缪尔 (J. Muir)强调自然的内在精神价值要高于其可以触摸 的物质价值, 提出了自然内禀价值(intrinsic value) 的概念，强调无人类干预的纯自然保护 (https://en.wikipedia.org/wiki/John_Muir)。平肖 (G. Pinchot)是美国林务局的首任局长，他提出了资源 保护伦理思想, 认为自然资源的适度利用就是在最 长的时间内让最多的人获得最大的利益, 资源应当 在个体间以及当代和后代间公平分配; 而且自然资 源应当被有效利用而不是白白地浪费 (https://en.wikipedia.org/wiki/Gifford_Pinchot)。20世 纪初叶, 利奥波德(A. Leopold)提出了保护的目标 是维护自然生态系统和生态过程的健康, 是人与自 然的和谐状态, 并成功地说服政府建立原野保护地 (wilderness area)。他认为人类是生态系统的一部分, 而不仅仅是自然资源的利用者。他提出的土地伦理 观点强调, 要改变人类作为土地系统征服者的角 色, 使其成为该系统的普通成员, 与土壤、水、植 物和动物等其他成员处于同等地位 (Leopold, 1949)。利奥波德被称为美国野生动物保护之父, 其 代表作为与 《瓦尔登湖》齐名的《沙乡年鉴》。20 世纪初叶的美国总统罗斯福(T. Roosevelt)对自然保 护的贡献功不可没, 他建立了国家林务局和第一个

\footnotetext{
* 通讯作者 Author for correspondence. E-mail: kpma@ibcas.ac.cn
} 
野生动物保护区以及其他数十个自然保护地。

伴随着保护观点和理论的形成和传播, 以及人 们对环境污染 (Carson,1962) 和人口快速增长 (Ehrlich,1968) 的担忧, 众多保护组织成立, 包括 1948年成立的世界自然保护联盟(IUCN)等, 一些保 护法案通过, 国家公园(如1872年建立的黄石国家 公园)和州公园等自然保护地得以建立, 大量的论 文著作发表。保护运动的蓬勃发展, 催生了保护生 物学 (Conservation Biology) 和保护生态学 (Conservation Ecology)两大学科。

保护生物学是关于生物多样性保护的科学, 带 有应对危机和重视实践的学科特点。然而, “保护生 物学”一词的出现早于“生物多样性”一词。Errington 和 Hamerstrom (1937) 在描述对环颈雉 (Phasianis colchicus) 的保护时首先使用了 “保护生物学” 这个 名词。当时主要是指对有经济价值的兽类和鸟类等 狩猎动物(game species)的保护。20世纪60-70年代, 人们不再仅仅关注经济物种的保护, 保护范围扩大 到全部物种, 即多样性(Pimlott,1969)。70年代后期, “保护生物学”一词经常出现在文献中。1978年第一 届国际保护生物学会议在美国的圣地亚哥举行, 对 于保护生物学而言这是具有标志性的事件。1985年 第二届会议时成立了国际保护生物学协会, 并决定 创办Conservation Biology杂志。1986年, 在美国举 行了国家生物多样性论坛, 在这次论坛之后, Wil$\operatorname{son}(1988)$ 主编的《生物多样性》一书出版, 在学术 界和国际社会产生了较大的影响。1987年 Conservation Biology杂志在美国创刊, 是保护生物 学领域创办的第二个旗舰刊物。第一个是1968年在 英国创办的 Biological Conservation。Biological Conservation 创刊号明确了刊物的定位, 提出了30 个重点方向, 反映了保护生物学研究的核心内容 (Harcourt, 1992)。虽然在这篇关于刊物定位的文章 中没有出现保护生物学一词, 但对学科发展的贡献 是显而易见的。

早期的保护行动大多以物种为对象, 而 Cox (1969)强调保护的生态系统途径, 提出“保护生态 学” 的概念。保护生态学概念的提出深受当时生态 系统生态学快速发展的影响。他在其主编的 Readings in Conservation Ecology一书的前言中写 道: “今天保护运动的最重要趋势是人们认识到保 护方针和实践必须依赖于坚实的生态学理论, 必须
致力于完整的生态系统的管理, 并由此产生了保护 生态学”。该书包括四个部分: (1)解决保护问题的生 态系统途径; (2)与生态系统能量流动相关的保护问 题; (3)与生态系统养分循环相关的保护问题; (4)自 然保护伦理。Cox (1993)认为保护生态学旨在获取 必要的生态学知识以保护和明智地利用生物多样 性, 是一个兼具基础性和应用性的学科。在这本唯 一的保护生态学教科书中, 他首先介绍了生态系 统、保护生态学和灭绝生态学; 接着分别讨论了陆 地和水域生态系统及其保护问题; 继而介绍了保护 生态学的理论和实践; 最后总结了自然保护的历史 和现状。Conservation Ecology杂志于1997年创刊, 是早期开放获取的在线刊物; 为了加强人文社会科 学的内容, 2004年改名为 Ecology and Society。 Science杂志至今仍设有“Conservation Ecology”栏目 (Phalan et al, 2016)。文献中经常会见到保护生态学 研究的表述, 但大多不是从学科出发, 而是为了保 护而开展的生态学研究。

1995年可以认为是生物多样性科学的起点, 主 要基于两个来源(马克平, 2016), 即两个相关的国际 计划: 2000年生物系统学议程(Systematics Agenda 2000)和国际生物多样性计划(DIVERSITAS)。生物 多样性科学与保护生物学和保护生态学稍有不同, 除同样重视解决生物多样性保护问题外, 更加强调 保护的科学基础, 如生物多样性的起源和维持机 制、分类和编目、变化和监测等方面(Loreau et al, 1999)。DIVERSITAS对于生物多样性科学的发展起 到了积极的推动作用, 2001年开始实施的第二个十 年计划, 包括生物起源(bioGENESIS)、生物发现 (bioDISCOVERY)、生态服务(ecoSERVICES)和生物 可持续性(bioSUSTAINABILITY) 4 个核心项目和农 业生物多样性(agroBIODIVERSITY)、淡水生物多 样性 (freshwaterBIODIVERSITY)、生态健康 (ecoHEALTH) 和全球山地生物多样性评估(GMBA) 4 个交叉科学计划(中国科学院生物多样性委员会, 2013)。未来的研究特别强调生物多样性格局、起源 和变化及其驱动因素, 生物多样性保护的社会和生 态科学基础, 生物多样性与生态系统服务对全球变 化的响应等方面(Larigauderie et al, 2012)。

文中参考文献见http://www.biodiversity-science. net/fileup/PDF/2016058-1.pdf。 


\section{参考文献}

Baeyens G, Martinez ML (2008) Coastal Dunes: Ecology and Conservation. Springer-Verlag, Berlin, Heidelberg.

Biodiversity Committee of the Chinese Academy of Sciences (2013) Translations on Biological Diversity (5). China Forestry Publishing House, Beijing. [中国科学院生物多样性委 员会 (2013) 生物多样性译丛(五). 中国林业出版社, 北 京.]

Carson R (1962) Silent Spring. Houghton Mifflin, Boston.

Cox GW (1969) Readings in Conservation Ecology. Appleton Century Crofts, New York.

Cox GW (1993) Conservation Ecology: Biosphere and Biosurvival. Brown (William C) Co., Dubuque.

Ehrlich PR (1968) Population Bomb. Ballantine Books, New York.

Errington PL, Hamerstrom FN (1937) The evaluation of nesting losses and juvenile mortality of the ring-necked pheasant. Journal of Wildlife Management, 1, 3-20.

Harcourt AH (1992) Conservation biology: its origins and definition. Science, 255, 1625.

Larigauderie A, Prieur-Richard AH, Mace GM, Lonsdale M, Mooney HA, Brussaard L, Cooper D, Cramer W, Daszak P, Díaz S, Duraiappah A, Elmqvist T, Faith DP, Jackson LE, Krug C, Leadley PW, Le Prestre P, Matsuda H, Palmer M, Perrings C, Pulleman M, Reyers B, Rosa EA, Scholes RJ, Spehn E, Turner II BL, Yahara T (2012) Biodiversity and ecosystem services science for a sustainable planet: the DIVERSITAS vision for 2012-20. Current Opinion in Envi- ronmental Sustainability, 4, 101-105.

Leopold A (1949) A Sand County Almanac. Oxford University Press, Oxford \& New York.

Loreau M, Olivieri I (1999) Diversitas: an international programme of biodiversity science. Trends in Ecology and Evolution, 14, 2-3.

Ma KP (2016) Hot topics for Biodiversity Science. Biodiversity Science, 24,1-2. (in Chinese) [马克平 (2016) 生物多样性 科学的热点问题. 生物多样性, 24, 1-2.]

Phalan B, Green RE, Dicks LV, Dotta G, Feniuk C, Lamb A, Strassburg BBN, Williams DR, Ermgassen E, Balmford A (2016) How can higher-yield farming help to spare nature? Science, 351, 450-451.

Pimlott DH (1969) The value of diversity. Transactions of the North American Wildlife and Natural Resources Conference, 34, 265-280.

Primack RP, Ma KP, Jiang ZG (2014) Essentials of Conservation Biology. Science Press, Beijing. (in Chinese) [Primack $R P$, 马克平, 蒋志刚 (2014) 保护生物学. 科学出版社, 北京.]

Stebbing EP (1922) The Forests of India, Vol. 1. John Lane, London.

Thoreau HD (translated by Xu C) (1982) Walden Pond. Shanghai Translation Publishing House, Shanghai. [亨利·大 卫.梭罗(徐迟译) (1982) 瓦尔登湖. 上海译文出版社, 上 海.]

Wilson EO (1988) Biodiversity. National Academy Press, Washington, DC. 